\title{
The contribution of collective prosumers to the energy transition in Europe - Preliminary estimates at European and country-level from the COMETS inventory
}

Authors: Valeria Jana Schwanitz, August Wierling, Jan Pedro Zeiss, Constantin von Beck, Ingrid Knutsdotter Koren, Timothy Marcroft, Lukas Müller, Melake Getabecha, Simon Dufner

Institute: Western Norway University of Applied Sciences

Corresponding author: Valeria Jana Schwanitz, valerias@hvl.no

Last update: August 21, 2021

\begin{abstract}
We present preliminary estimates for the contribution of collective prosumers to the energy transition across Europe. Drawing from the COMETS inventory, we report country-level and EU-level aggregates for current total numbers of initiatives and their historic evolution, the fields of engagement, the number of people involved, renewable capacities installed, number of projects administered, and total funds invested. Currently, at least two million citizens across Europe collectively engage in more than 8400 energy communities, realizing a minimum of 13000 projects and investing at least 2.6 billion EUR.
\end{abstract}

\section{Introduction}

The successful transition of national energy systems from fossil-fuel based systems to renewable-based systems benefits from the engagement of citizens in transformation processes in various ways. Across Europe, citizens form different types of collective action initiatives (CAI) to invest in renewable energy projects, to ramp up renewable capacities, or to motivate and support others in participating. In these ways, citizens act as pioneers and explorers of the necessary system changes. They test the practicability of technical and legislative solutions, experiment for the benefit of the local community, serve as partners to public authorities in transition initiatives, identify new business models, and, most importantly, they partake in the discussions about the shape of the future energy system.

Case studies of CAI have been carried out in many countries. However, aggregate quantitative information is largely lacking (c.f. Wierling et al. 2018, Berka et al. 2018). As a response to these missing aggregates, this paper presents the first systematic insights and aggregates, drawing from an inventory that has been compiled over the last 3 years, as part of the EU funded project "COMETS - Collective Action Models for the Energy Transition and Social Innovation" (05/2019-04/2022). We report preliminary country-level and EU-level aggregates for current total numbers of initiatives and their historic evolution, fields of engagement, number of people involved, renewable capacities installed, number of projects administered, and total funds invested. The focus of the paper is on CAls with legal registration. Projects without legal registration are also recorded if possible, but no systematic search has been made.

It is important to note that this paper presents preliminary estimates only. A final update and open publication of the inventory on collective action initiatives in the energy transition is planned for Spring 2022.

\section{Context}

Members and associated countries of the European Union are currently implementing EU Directives 2018/2001 and 2019/944 which define the terms "Renewable energy community" and "Citizen energy community", respectively. Both legal terms also outline the space for what we 
operationalize in the COMETS inventory as collective action initiatives (CAI). While some differences between these three concepts exist, the important points are that:

- Members participate voluntarily and have full control over their initiatives (e.g., following the 'One-member, one-vote' rule as is common among energy cooperatives),

- The initiative benefits the local community (socially, environmentally, and/or economically).

The focus of the paper is on collective action initiatives that engage in the sustainable energy transition. This means that we compile data on citizen-led energy projects that involve renewable energy, energy saving, sustainable energy consulting, and services. We do not cover initiatives that solely engage with fossil-fuels (e.g., gas or conventional heating networks).

\section{Materials and methods}

The results are based on the COMETS inventory on collective action initiatives in the energy transition. The data has been compiled over the last 3 years, as part of the COMETS project. Main data sources include national business and trade registries, web pages of initiatives and network associations, annual reports, and media coverage. Current publications on the COMETS database include Wierling et al. 2018, Wierling et al. 2020, Wierling et al. 2021 a, Wierling et al. 2021 b (submitted), Beck 2021, Schwanitz et al. 2021 (submitted). These papers provide further information on data collection methods and sources. A final update with comprehensive documentation of the inventory on collective action initiatives in the energy transition is planned for Spring 2022. The data will be open access.

\section{Preliminary results}

\section{European-level results}

Currently, at least two million ${ }^{1}$ citizens across Europe collectively engage in more than 8400 energy communities ${ }^{2}$, realizing a minimum of 13000 projects $^{3}$ since the year 2000 . Pioneering the installation of renewable capacities in Denmark and other countries, they support the energy transition and contribute to the competitiveness of renewable energy technologies in various ways. Energy communities raise technology awareness and acceptance, promote energy efficiency, produce and distribute renewable-based electricity, provide services around e-mobility, and run energy consulting services. They experiment innovatively with business models and self-sufficiency concepts for the benefit of local communities.

Fig. 1 details the number of initiatives and projects per country. Differences across countries can be explained by the strength of governmental support and incentives schemes, historic path dependencies of the energy system, and social and technological preferences. Current total renewable capacities installed by citizen-led energy projects in Europe can be estimated at least as high as $6.3 \mathrm{GW}^{4}$, contributing up to $7 \%$ to the nationally installed capacities ${ }^{5}$. The lion's share is

\footnotetext{
${ }^{1}$ Based on numbers from 15 countries. Information is missing for CYP, LUX, SLV; NOR; IRE, ITA, HUN, LVA, ROM, PRT, ESP, GRC, MLT, BGR, LIE, SVK.

${ }^{2}$ Based on numbers from CAls from 28 countries, missing information for CYP, LIE, and SLK.

${ }^{3}$ Based on numbers for 19 countries, missing information for LUX, IRE, BGR, CYP, GRC, ESP, PRT, ROM, SLK, HUN, LVA, LIE.

${ }^{4}$ Based on numbers from 18 countries, excluding terminated projects. Missing information for LUX, BGR, CYP, GRC, HUN, IRE, LVA, LIE, PRT, ROM, SLK, CHE, ESP.

${ }^{5}$ Using the Belgium estimate (highest value).
} 
taken by solar PV $(\sim 50 \%)^{6}$, followed by onshore wind $(\sim 10 \%){ }^{7} \mathrm{~A}$ conservative estimate of the total invested finances amounts to at least 2.6 billion EUR. ${ }^{8}$

The continuation and extension of energy communities in Europe depends on favorable legislative and financial incentives as well as on the competitiveness of technologies that are accessible to citizens. The latter is in particular the case for granular renewable technologies, such as roof-top solar, small- to medium-size wind and solar parks, heat pumps in private and cooperative housing, micro hydro, biomass furnaces, and biogas installations.

The European level aggregates are preliminary, rough estimates based on the current state of the COMETS database. Country-level results below have been aggregated for this purpose (see following tables). In case no data was available, the contribution of that country to the total estimate was not accounted for. Therefore, the estimates can be seen as conservative, lower estimates.

Fig. 1: Collective action initiatives in Europe (8431). The figure presents preliminary estimates based on the COMETS database. Shown are the number of initiatives in a country. The color code for the countries shows the civil society participation index of the Varieties of Democracies Project VDEM (Coppedge et al. 2021).

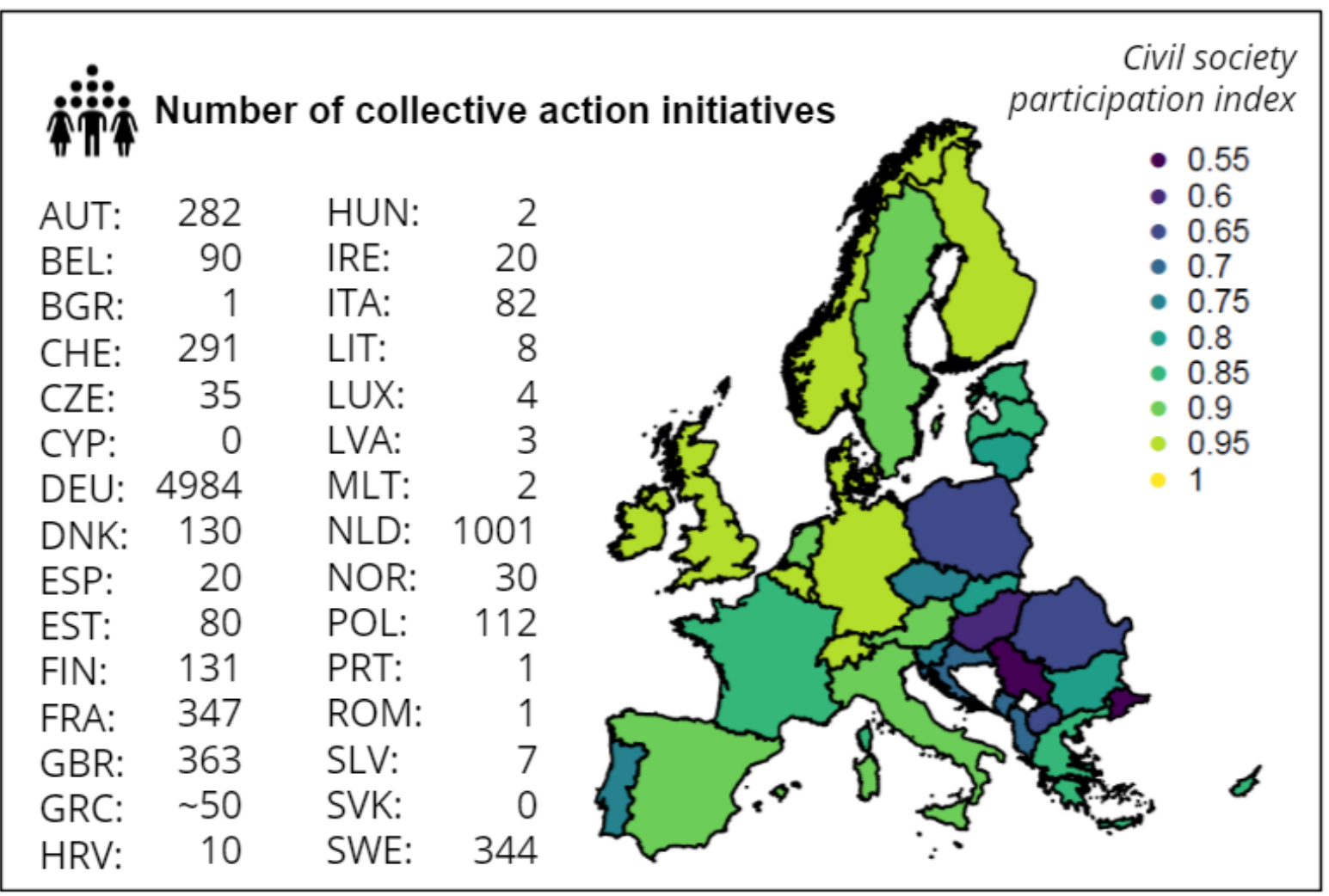

\footnotetext{
${ }^{6}$ Based on data from 12 countries, not counting terminated and planned initiatives as well as initiatives that do not have their primary focus on the energy sector (e.g., agricultural initiatives).

${ }^{7}$ Counting the contribution from seven countries, excluding terminated and planned initiatives as well as initiatives not having a primary focus on the energy sector.

${ }^{8}$ Estimate based on data from 10 countries.
} 


\section{Preliminary country-level results}

\section{Austria}

\begin{tabular}{|l|l|l|}
\hline Essential information & Quantitative estimate & Notes \\
\hline $\begin{array}{l}\text { Current total number of } \\
\text { initiatives }\end{array}$ & 282 cooperatives & Typical forms: cooperatives, associations. \\
\hline $\begin{array}{l}\text { Historical evolution of } \\
\text { number of initiatives }\end{array}$ & Slow increase since late 1980s. & Wierling et al. 2018 \\
\hline Field of engagement & $\begin{array}{l}95 \% \text { of coops are active in district } \\
\text { heating with biomass. }\end{array}$ & $\begin{array}{l}\text { Early engagement in wind energy, solar PV only } \\
\text { emerging. }\end{array}$ \\
\hline Number of people involved & $>564$ & Min. \# of members per CAl: 2 \\
\hline $\begin{array}{l}\text { Renewable capacities } \\
\text { installed }\end{array}$ & Wind ( 400-500 MW) & $\begin{array}{l}\text { No update yet available. Estimate for 2010 } \\
\text { based on Wierling et al. 2018. No aggregate } \\
\text { data for heating yet. }\end{array}$ \\
\hline Number of projects & $>282$ & Assuming 1 project per CAl. \\
\hline Total funds invested & n/a & n/a \\
\hline
\end{tabular}

\section{Belgium}

\begin{tabular}{|l|l|l|}
\hline Essential information & Quantitative estimate & Notes \\
\hline $\begin{array}{l}\text { Current total number of } \\
\text { initiatives }\end{array}$ & 90 & $\begin{array}{l}\text { We expect about 20 more initiatives to be } \\
\text { added to the database. The most common } \\
\text { forms are 3 types of cooperatives. }\end{array}$ \\
\hline $\begin{array}{l}\text { Historical evolution of } \\
\text { number of initiatives }\end{array}$ & $\begin{array}{l}\text { Peak in 2017, strong recent } \\
\text { development. }\end{array}$ & $\begin{array}{l}\text { Good governmental support, but a variety of } \\
\text { regional specifications. }\end{array}$ \\
\hline Field of engagement & $\begin{array}{l}\text { Solar PV (11\%), wind (88\%), heat, } \\
\text { others. }\end{array}$ & Estimate based on installed capacities. \\
\hline Number of people involved & $\sim 120000 ;$ one big with $~ 60.000$. & Typical size: <600, few >1000 members. \\
\hline $\begin{array}{l}\text { Renewable capacities } \\
\text { installed }\end{array}$ & $\begin{array}{l}\text { Solar PV: 39 MW (7\% of national } \\
\text { installed PV), wind (7\% of nationally } \\
\text { installed capacities): 330 MW. }\end{array}$ & Based on the COMETS database. \\
\hline $\begin{array}{l}\text { Number of projects } \\
\text { administered }\end{array}$ & 576 & Database entries. \\
\hline Total funds invested & n/a & Upcoming. \\
\hline
\end{tabular}

\section{Croatia}

\begin{tabular}{|l|l|l|}
\hline Essential information & Quantitative estimate & Notes \\
\hline $\begin{array}{l}\text { Current total number of } \\
\text { initiatives }\end{array}$ & $\sim 10$ & $\begin{array}{l}\text { Total number based on an interview with Edo } \\
\text { Jerkic from ZEZ in 2019.8 cooperatives in the } \\
\text { COMETS database. }\end{array}$ \\
\hline $\begin{array}{l}\text { Historical evolution of } \\
\text { number of initiatives }\end{array}$ & Starting in 2013, so far peak 2018. & $\begin{array}{l}\text { The United Nations Development Program } \\
\text { initiated “Energy cooperatives in Croatia" in } \\
\text { 2013. The sector is expected to grow. }\end{array}$ \\
\hline
\end{tabular}




\begin{tabular}{|l|l|l|}
\hline Field of engagement & $\begin{array}{l}\text { 1 wind farm operated by a } \\
\text { cooperative bank, 3 PV systems. }\end{array}$ & $\begin{array}{l}\text { Based on a presentation from Jerkic 2016. A } \\
\text { variety of single projects as testbeds (e.g., wind, } \\
\text { rooftop solar PV, biomass/biogas for heating, } \\
\text { e-mobility, crowdfunding, consultation). }\end{array}$ \\
\hline Number of people involved & $\sim 1300$ & $\begin{array}{l}\text { Information on members only available for two } \\
\text { cooperatives: } \\
\text { ZEZ (18 members) and ZEF (1200); a } \\
\text { cooperative for ethical financing. Estimate } \\
\text { based on the smaller CAl as the more } \\
\text { representative one. }\end{array}$ \\
\hline $\begin{array}{l}\text { Renewable capacities } \\
\text { installed }\end{array}$ & $<10.5 \mathrm{MW}$ (thereof: 10.3 MW wind) & $\begin{array}{l}\text { + up to 50 MW planned/feasibility study stage. } \\
\text { entries. }\end{array}$ \\
\hline Number of projects & $8-10$ (2016) & Jerkic (2016). \\
\hline Total funds invested & $\begin{array}{l}63800 \mathrm{EUR}(\mathrm{PV}), 21.74 \text { million EUR } \\
\text { (wind) }\end{array}$ & $\begin{array}{l}\text { Number from two initiatives where investments } \\
\text { are known. }\end{array}$ \\
\hline
\end{tabular}

\section{Czech Republic}

\begin{tabular}{|c|c|c|}
\hline Essential information & Quantitative estimate & Notes \\
\hline $\begin{array}{l}\text { Current total number of } \\
\text { initiatives }\end{array}$ & 35 (potentially up to 110 ). & $\begin{array}{l}\text { Mostly agricultural, housing, and construction } \\
\text { coops. }\end{array}$ \\
\hline $\begin{array}{l}\text { Historical evolution of } \\
\text { number of initiatives }\end{array}$ & $\begin{array}{l}\text { Peaks in the } 1950 \text { s and } 90 \text { s. } 2 \text { new } \\
\text { coops after } 2000 .\end{array}$ & Implementing EU Directives on its way. \\
\hline Field of engagement & $\begin{array}{l}\text { Biomass/biogas ( } 82 \%) \text {, solar } \\
\text { thermal }(11 \%) \text {, solar PV ( } 5 \%) \text {. }\end{array}$ & $\begin{array}{l}\text { Agricultural/housing/construction } \\
\text { cooperatives. } 1 \text { planned windpark. }\end{array}$ \\
\hline Number of people involved & 17000 (up to 53000 ). & $\begin{array}{l}\text { Upper estimate based on one share per } \\
\text { member. }\end{array}$ \\
\hline $\begin{array}{l}\text { Renewable capacities } \\
\text { installed }\end{array}$ & $\begin{array}{l}\text { Heat: } 21 \mathrm{MW} \text { (potentially up to } 67 \\
\mathrm{MW} \text { ), electricity: } 22 \mathrm{MW} \text { (potentially } \\
\text { up to } 69 \mathrm{MW} \text { ). }\end{array}$ & Capacity from planned wind park not included. \\
\hline Number of projects & 35 (potentially up to 110 ). & Typically 1 project per CAI. \\
\hline Total funds invested & $\begin{array}{l}<15.5 \text { million EUR (potentially up } \\
\text { to } 48,6 \text { million EUR). }\end{array}$ & $\begin{array}{l}\text { Estimated from shares. CAls have a secondary } \\
\text { focus to be financed, thus the estimate is upper } \\
\text { limit. }\end{array}$ \\
\hline
\end{tabular}

\section{Denmark}

\begin{tabular}{|l|l|l|}
\hline Essential information & Quantitative estimate & Notes \\
\hline $\begin{array}{l}\text { Current total number of } \\
\text { initiatives }\end{array}$ & 130 & $\begin{array}{l}\text { Just 12 \% of the former 1109 wind coops exist } \\
\text { (Wierling et al., 2018). }\end{array}$ \\
\hline $\begin{array}{l}\text { Historical evolution of } \\
\text { number of initiatives }\end{array}$ & $\begin{array}{l}\text { Two waves of foundation of wind } \\
\text { cooperatives in Denmark } \\
(1985-1992 \text { and 1998-2002). } \\
\text { Strong decrease from 2002. }\end{array}$ & $\begin{array}{l}\text { Change in FIT from 2002 and beginning of } \\
\text { commercial offshore wind investments. Coops } \\
\text { driving onshore wind were not able to continue } \\
\text { with offshore wind. }\end{array}$ \\
\hline Field of engagement & Onshore wind, solar PV, others. & Private data restrictions. \\
\hline
\end{tabular}




\begin{tabular}{|l|l|l|}
\hline Number of people involved & 5000-0.5 million & Domination of smaller coops, 5-200 members. \\
\hline $\begin{array}{l}\text { Renewable capacities } \\
\text { installed }\end{array}$ & Wind (340-600 MW) & $\sim 15-40 \%$ of turbines are/were owned by coops. \\
\hline Number of projects & Around 1109. & Assuming one project per initiative. \\
\hline Total funds invested & Up to 400 million EUR. & Rough estimate. Figures missing. \\
\hline
\end{tabular}

\section{Estonia}

\begin{tabular}{|l|l|l|}
\hline Essential information & Quantitative estimate & Notes \\
\hline $\begin{array}{l}\text { Current total number of } \\
\text { initiatives }\end{array}$ & 131 & $\begin{array}{l}96 \% \text { apartment associations, 1 ecovillage, } 1 \\
\text { NPO, other business). }\end{array}$ \\
\hline $\begin{array}{l}\text { Historical evolution of } \\
\text { number of initiatives }\end{array}$ & n/a & $\begin{array}{l}\text { Emerging sector. Establishment of support } \\
\text { programs under development. }\end{array}$ \\
\hline Field of engagement & Solar PV, solar thermal. & Installed on apartment houses. \\
\hline Number of people involved & $393-1300$ & $\begin{array}{l}\text { Assumption: 3-10 units per apartment house } \\
\text { with one member per unit. }\end{array}$ \\
\hline $\begin{array}{l}\text { Renewable capacities } \\
\text { installed }\end{array}$ & $2740-6550$ kW & $\begin{array}{l}\text { Based on the average size of solar PV } \\
\text { installations in housing coops (20-50 kWp). }\end{array}$ \\
\hline Number of projects & 137 production units. & Database entries \\
\hline Total funds invested & $\sim 1-1.4$ mill EUR. & $\begin{array}{l}\text { Estimate: subsidized reconstruction and } \\
\text { investment grants. }\end{array}$ \\
\hline
\end{tabular}

\section{Finland}

\begin{tabular}{|l|l|l|}
\hline Essential information & Quantitative estimate & Notes \\
\hline Current number of initiatives & 77 entries (potentially: 80-120). & Cooperatives are the most common form \\
\hline $\begin{array}{l}\text { Historical evolution of } \\
\text { number of initiatives }\end{array}$ & Few new CAls after 2010. & $\begin{array}{l}\text { Earliest cooperatives established in } 1918 \\
\text { (electricity grid operation). }\end{array}$ \\
\hline Field of engagement & $\begin{array}{l}\text { Heating (biomass, oil): } 47 \% ; \\
\text { el-distribution: } 20 \% ; \text { Hydropower: } \\
\sim 9 \% \text { Wind, solar-PV, consulting. }\end{array}$ & Based on database entries. \\
\hline Number of people involved & $\begin{array}{l}112000 \text { (potentially up to 117000). } \\
\text { Only four cooperatives with member } \\
\text { information. Between 50-103420 members. } \\
\text { Estimate based on categorization of CAls } \\
\text { along yearly turnover, assuming higher } \\
\text { turnover indicates larger member numbers. }\end{array}$ \\
\hline $\begin{array}{l}\text { Renewable capacities } \\
\text { installed }\end{array}$ & $\begin{array}{l}\text { Electricity: 32-133 MW (potentially up } \\
\text { to 210 MW); heat: 137-367 MW (up } \\
\text { to } 560 \text { MW). }\end{array}$ & $\begin{array}{l}\text { Low estimate excludes outliers, high estimate } \\
\text { includes all. Not included: } 29 \text { TWh/a RE-based } \\
\text { heat and electricity from the largest coop } \\
\text { ( 15\% of national RE production). }\end{array}$ \\
\hline Number of projects & $\begin{array}{l}150 \text { (potentially up to 240). } \\
\text { n/a funds invested }\end{array}$ & $\begin{array}{l}\text { Assumption: 3 projects/CAl and 65\% of CAls } \\
\text { being active in energy production (based on } \\
\text { collected data). Largest coop as outlier. }\end{array}$ \\
\hline
\end{tabular}


France

\begin{tabular}{|c|c|c|}
\hline Essential information & Quantitative estimate & Notes \\
\hline $\begin{array}{l}\text { Current total number of } \\
\text { initiatives }\end{array}$ & $\begin{array}{l}347 \text { (primary focus on energy market, } \\
\text { excluding CAl engaged in general in } \\
\text { energy transition) }\end{array}$ & $\begin{array}{l}\text { Many legal forms in France, incl. citizen } \\
\text { energy projects, associations, Local } \\
\text { Distribution Companies, coops. }\end{array}$ \\
\hline $\begin{array}{l}\text { Historical evolution of } \\
\text { initiatives }\end{array}$ & $\begin{array}{l}\text { New CAl creations peaked in } 2018, \\
\text { declining in } 2019 \text { \& } 2020 .\end{array}$ & Reductions in FiT, possible COVID-19 effect. \\
\hline Field of engagement & $\begin{array}{l}\text { Solar PV ( } 54 \%) \text {, energy education } \\
(46 \%) \text {, onshore wind ( } 8 \%) \text {, microhydro } \\
(3 \%) \text {, biomass (3\%), biogas ( } 2 \%) \text {. }\end{array}$ & $\begin{array}{l}\text { Almost all productive CAls specialize in one } \\
\text { technology, and many participate as well in } \\
\text { education and consciousness raising. }\end{array}$ \\
\hline Number of people involved & $\begin{array}{l}\text { 130,000 (thereof about: 55,000 } \\
\text { Enercoop, 40,000 la Nef Cooperative } \\
\text { Bank, 18,000 currently producing } \\
\text { CAls, 8,000 other and developing } \\
\text { CAls, 7,000 local distribution } \\
\text { companies, 2,000 mobility). }\end{array}$ & $\begin{array}{l}\text { Figure based on representative sample of } \\
\text { each category with mean taken from second } \\
\text { and third quartiles and applied to all CAls } \\
\text { with no membership number available. } \\
\text { Figure excludes } \sim 100 \text { associations, most with } \\
\text { no listed member figures. }\end{array}$ \\
\hline $\begin{array}{l}\text { Renewable capacities } \\
\text { installed }\end{array}$ & $\begin{array}{l}\sim 205 \mathrm{MW} \text { installed, } \sim 210 \mathrm{MW} \text { planned } \\
\text { identified (ca. } 0.35 \% \text { of French } \\
\text { installed RE capacity). }\end{array}$ & $\begin{array}{l}\text { Based on units in the COMETS database. } \\
\text { Excluding } 5 \text { units without data. }\end{array}$ \\
\hline Number of projects & $\begin{array}{l}\sim 760 \text { installed, } \sim 200 \text { planned } \\
\text { installments identified }\end{array}$ & $\begin{array}{l}\text { Based on units in the database, aggregates } \\
\text { are assumed to have } 3 \text { units. }\end{array}$ \\
\hline Total funds invested & $\begin{array}{l}\text { 154-176 million EUR (thereof: } 33 \\
\text { MEUR PV, } 96 \text { MEUR wind, } 10 \text { MEUR } \\
\text { biogas, } 9 \text { MEUR wood, } 9 \text { MEUR heat, } \\
\text { hydro } 6 \text { MEUR hydro). }\end{array}$ & $\begin{array}{l}\text { Low estimate excludes units with unknown } \\
\text { ownership. High estimate assumes equal } \\
\text { shares. Solar PV (biogas) estimates from IEA } \\
\text { country reports (ADEME figures) if n/a. Other } \\
\text { technologies are means of investment cost.. }\end{array}$ \\
\hline
\end{tabular}

Germany

\begin{tabular}{|c|c|c|}
\hline Essential information & Quantitative estimate & Notes \\
\hline $\begin{array}{l}\text { Current total number of } \\
\text { initiatives }\end{array}$ & $\begin{array}{l}1550 \text { coops with primary purpose } \\
\text { 'energy transition (ET)', up to } \\
\text { 3012-3434 associations owning RE } \\
\text { production units or engaging in ET. }\end{array}$ & $\begin{array}{l}\text { Typical legal forms: coops, associations. } \\
\text { Majority of associations do not have a } \\
\text { primary focus on energy transition. }\end{array}$ \\
\hline $\begin{array}{l}\text { Historical evolution of } \\
\text { number of initiatives }\end{array}$ & $\begin{array}{l}\text { Peak in } 2014,2 / 3 \text { continue investments } \\
\text { thereafter. }\end{array}$ & $\begin{array}{l}\text { Change in FiT. Associations started to engage } \\
\text { in ET since around } 2000 \text { and recently. }\end{array}$ \\
\hline Field of engagement & $\begin{array}{l}\text { Solar PV }(81 \%) \text {, onshore wind }(13 \%) \text {, } \\
\text { hydro }(0.6 \%) \text {, biomass }(4.8 \%) ; \\
\text { associations: majority solar PV. }\end{array}$ & $\begin{array}{l}\text { Numbers are based on coop projects with } \\
\text { primary focus on energy transition. }\end{array}$ \\
\hline Number of people involved & Energy coops: $\sim 300.000$ & $\begin{array}{l}\text { Unspecified for associations which may } \\
\text { range between } 7 \text { to several hundred of } \\
\text { members. }\end{array}$ \\
\hline $\begin{array}{l}\text { Renewable capacities } \\
\text { installed }\end{array}$ & $\begin{array}{l}\text { Up to } 3 \mathrm{GW} \text {; Solar PV: } 600-848 \mathrm{MWp} \text {; } \\
\text { wind: } 1-1.9 \mathrm{GW} \text {; hydro: } 6.4 \mathrm{MW} \text {, } \\
\text { biomass/heat: } 110 \mathrm{MW} \text {; altogether } \\
\sim 2-3 \% \text { of national capacities (thereof: } \\
\text { solar PV 1.2-1.7\%; wind: 3.5\%). }\end{array}$ & $\begin{array}{l}\text { Based on Wierling et al. } 2021 \text { and COMETS } \\
\text { database updates. }\end{array}$ \\
\hline Number of projects & $\begin{array}{l}\sim 4530 \text { (ca. } 3672 \text { PV projects, } 597 \text { wind, } \\
28 \text { hydro, biomass: } 216 \text { ), associations: }\end{array}$ & $\begin{array}{l}\text { Estimate from coops with primary purpose } \\
\text { on energy transition; }\end{array}$ \\
\hline
\end{tabular}




\begin{tabular}{|l|l|l|}
\hline & $\begin{array}{l}\sim 4129 \text { PV projects; } 6 \text { wind turbines } \\
\text { and 159 storage projects. Total } \\
\text { number of projects (primary \& } \\
\text { secondary focus): } ~ 10000 .\end{array}$ & $\begin{array}{l}\text { Indication of size/complexity: Wind farm } \\
\text { projects may differ between 1-36 turbines. }\end{array}$ \\
\hline Total funds invested & $\begin{array}{l}\text { Coops: } \sim 0.8 \text { billion EUR; associations: } \\
\sim 0.36 \text { billion EUR }\end{array}$ & $\begin{array}{l}\sim 0.36 \text { billion EUR (thereof: solar PV } \sim 0.35 \\
\text { billion EUR, storage } ~ \\
<0.005 \text { billion EUR, wind }\end{array}$ \\
\hline
\end{tabular}

\section{Great Britain}

\begin{tabular}{|l|l|l|}
\hline Essential information & Quantitative estimate & Notes \\
\hline $\begin{array}{l}\text { Current total number } \\
\text { of initiatives }\end{array}$ & 363 & $\begin{array}{l}\text { Forms: initiatives registered under the Co-op } \\
\text { and Community Benefit Societies Act. }\end{array}$ \\
\hline $\begin{array}{l}\text { Historical evolution of } \\
\text { number of initiatives }\end{array}$ & $\begin{array}{l}\text { First wave 2010-2015, declined from 2016. } \\
\text { Recently a new increase. }\end{array}$ & Mirroring FiT changes. \\
\hline Field of engagement & $\begin{array}{l}\text { Wind (14\%), solar PV (40\%), hydro (16\%); } \\
\text { energy consulting (20\%). }\end{array}$ & Based on Wierling et al. 2018. \\
\hline $\begin{array}{l}\text { Number of people } \\
\text { involved }\end{array}$ & $\sim 17000$ & $\begin{array}{l}300-750 \text { members per CAl. New initiatives } \\
\text { tend to have minimum numbers. }\end{array}$ \\
\hline $\begin{array}{l}\text { Renewable capacities } \\
\text { installed }\end{array}$ & Total: 150 MWp. & Wierling et al. 2018 \\
\hline Number of projects & $\sim 2800$ & $\begin{array}{l}\text { New initiatives start with just one project. } \\
\text { Later 5-10 projects per CAl. }\end{array}$ \\
\hline Total funds invested & $\begin{array}{l}800000 \text { GBP/CAl, total: 290.4 million GBP } \\
(\sim 339 \text { million EUR). }\end{array}$ & $\begin{array}{l}\text { From Wierling et al. 2018, based on 49 coops } \\
\text { revealing this information. }\end{array}$ \\
\hline
\end{tabular}

\section{Italy}

\begin{tabular}{|l|l|l|}
\hline Essential information & Quantitative estimate & Notes \\
\hline $\begin{array}{l}\text { Current total number of } \\
\text { initiatives }\end{array}$ & 82 & Coops are the common legal form. \\
\hline $\begin{array}{l}\text { Historical evolution of } \\
\text { initiatives }\end{array}$ & Few new CAl since 2011. & Depending on FiT. \\
\hline Field of engagement & Solar PV (94\%) & $\begin{array}{l}\text { Some hydro projects from historical energy } \\
\text { coops. 94\% with primary focus on solar. }\end{array}$ \\
\hline $\begin{array}{l}\text { Number of people } \\
\text { involved }\end{array}$ & n/a & n/a \\
\hline $\begin{array}{l}\text { Renewable capacities } \\
\text { installed }\end{array}$ & Solar PV (11 MWp) & Estimate from Wierling et al. 2021 \\
\hline Number of projects & 64 & Solar PV projects \\
\hline Total funds invested & n/a & n/a \\
\hline
\end{tabular}




\begin{tabular}{|c|c|c|}
\hline Essential information & Quantitative estimate & Notes \\
\hline Number of initiatives & 8 & Housing associations. \\
\hline $\begin{array}{l}\text { Historical evolution of } \\
\text { number of initiatives }\end{array}$ & $\mathrm{n} / \mathrm{a}$ & Emerging topic. \\
\hline Field of engagement & $\mathrm{n} / \mathrm{a}$ & $\begin{array}{l}\text { Renovating heating in housing (various technologies, } \\
\text { incl. geothermals, solar PV, biomass). }\end{array}$ \\
\hline Number of people & 160 & Members of housing and village associations. \\
\hline $\begin{array}{l}\text { Renewable capacities } \\
\text { installed }\end{array}$ & Solar (17 kWp), wind (250 kW) & Estimate based on BSR database (Co2mmunity 2021). \\
\hline Number of projects & 8 & Assuming at least one project/CAl. \\
\hline Total funds invested & $\sim 0.4$ Million EUR & $\begin{array}{l}\text { Solar ( } 17,000 \text { EUR, estimate based on capacity), wind } \\
\text { ( 0.4 million EUR from BSR database). }\end{array}$ \\
\hline
\end{tabular}

\section{Luxembourg}

\begin{tabular}{|l|l|l|}
\hline Essential information & Quantitative estimate & Notes \\
\hline $\begin{array}{l}\text { Current total number of } \\
\text { initiatives }\end{array}$ & 4 & Identified coops related to energy transition \\
\hline $\begin{array}{l}\text { Historical evolution of } \\
\text { number of initiatives }\end{array}$ & Emerging sector. & \\
\hline Field of engagement & $\begin{array}{l}\text { Solar PV, wood pellet heating, } \\
\text { Onshore Wind }\end{array}$ & No data \\
\hline $\begin{array}{l}\text { Number of people } \\
\text { involved }\end{array}$ & n/a & Ownership for wind is unclear. \\
\hline $\begin{array}{l}\text { Renewable capacities } \\
\text { installed }\end{array}$ & $\begin{array}{l}\text { Solar PV (60 kWp), heating } \\
(777000 \mathrm{kWh}), \text { wind (no data) })\end{array}$ & Database entries \\
\hline $\begin{array}{l}\text { Number of projects } \\
\text { administered }\end{array}$ & 7 & Only reported and realized investments. \\
\hline Total funds invested & 208000 & \\
\hline
\end{tabular}

\section{Netherlands}

\begin{tabular}{|l|l|l|}
\hline Essential information & Quantitative estimate & Notes \\
\hline $\begin{array}{l}\text { Current total number of } \\
\text { initiatives }\end{array}$ & $1001+/-5 \%$ & $\begin{array}{l}\text { Energy cooperatives, foundations, associations with } \\
\text { primary purpose 'energy transition'. Main source: } \\
\text { Hier Opgewekt (2021) }\end{array}$ \\
\hline $\begin{array}{l}\text { Historical evolution of } \\
\text { number of initiatives }\end{array}$ & $\begin{array}{l}\text { Ca. since 2015 (focus on wind), } \\
\text { peak in 2018, recently many } \\
\text { new initiatives. }\end{array}$ & $\begin{array}{l}\text { Based on the COMETS database. See also Hufen \& } \\
\text { Koppenjan (2015), Boon \& Dieperink (2014) }\end{array}$ \\
\hline Field of engagement & Solar PV (92\%), wind (8\%). & $\begin{array}{l}\text { Solar PV is also established on water. Another field of } \\
\text { engagement is also energy coaching, here not } \\
\text { included in the statistics. }\end{array}$ \\
\hline
\end{tabular}




\begin{tabular}{|c|c|c|}
\hline $\begin{array}{l}\text { Number of people } \\
\text { involved }\end{array}$ & $\sim 120,000$ & $\begin{array}{l}\text { Based on estimates obtained from a subset of } 280 \\
\text { CAl for which membership numbers are known. } \\
\text { About } 12 \text { outliers with members }>500 \text { are excluded } \\
\text { from the sample, but included in the final estimate. }\end{array}$ \\
\hline $\begin{array}{l}\text { Renewable capacities } \\
\text { installed }\end{array}$ & $\begin{array}{l}\text { Solar PV 140- } 205 \text { MW, (up to } \\
270 \text { MW with planned projects); } \\
\text { wind 237- } 264 \text { MW (up to } 1884 \\
\text { MW with planned projects). }\end{array}$ & $\begin{array}{l}\text { Low estimates: realized projects until end of 2020, } \\
\text { crowdfunding projects excluded, no ownership } \\
\text { assumed for projects with unclear ownership share. } \\
\text { High estimates: realized projects until end of } 2020 \text {, } \\
\text { includes crowdfunding, } 100 \% \text { ownership assumed for } \\
\text { projects with unclear ownership share. Brackets } \\
\text { show high estimate including planned projects) }\end{array}$ \\
\hline $\begin{array}{l}\text { Number of projects } \\
\text { administered }\end{array}$ & $\begin{array}{l}\sim 1442 \text { total (thereof: } 940 \\
\text { realized, } 473 \text { planned, } 14 \text { wind } \\
\text { repowering, } 15 \text { planned but } \\
\text { never realized). }\end{array}$ & Based on the COMETS database. \\
\hline Total funds invested & $\begin{array}{l}\text { Estimate 1: } \\
\text { PV: } 224 \text { - } 329 \text { million Euro } \\
\text { Wind: } 390 \text { - } 431 \text { million }\end{array}$ & $\begin{array}{l}\text { <Estimate for PV from Hier Opgewekt (2021): } \\
\text { 2012-2020: } 180-200 \text { billion EUR invested. Based on PV } \\
\text { solar capacity estimate of } 166 \text { MWp ( } 300 \text { EUR per } \\
\text { panel/275 Wp). } \\
\text { Estimate } 1 \text { (PV): Based on system prices for } \\
\text { installation of } 10 \text { Panels, including costs for modules, } \\
\text { installation, converters and VAT. Datasource for } \\
\text { prices: Milieu Centraal (2021). Estimate } 1 \text { (Wind): } \\
\text { based on average price of } 2000 \text { EUR/kw until 2013, } \\
\text { 1500 EUR/kw thereafter. Excludes planned projects. } \\
\text { Datasource for prices: Paardenkooper (2015), } \\
\text { Madlener et al. (2019). }\end{array}$ \\
\hline
\end{tabular}

\section{Norway}

\begin{tabular}{|c|c|c|}
\hline Essential information & Quantitative estimate & Notes \\
\hline $\begin{array}{l}\text { Current total number of } \\
\text { initiatives }\end{array}$ & $\sim 30(2018)$ & $\begin{array}{l}\text { Local Energy Communities. Driven by property } \\
\text { developers and distribution network operators. Data } \\
\text { is fully anonymized. }\end{array}$ \\
\hline $\begin{array}{l}\text { Historical evolution of } \\
\text { number of initiatives }\end{array}$ & $\begin{array}{l}\text { Recent developments, still in } \\
\text { their infancy. }\end{array}$ & \\
\hline Field of engagement & Under development. & $\begin{array}{l}\text { Solar PV, energy efficiency, end-user flexibility, } \\
\text { security of supply, grid investments. Lack of data. }\end{array}$ \\
\hline People involved & $\mathrm{N} / \mathrm{A}$ & \\
\hline $\begin{array}{l}\text { Renewable capacities } \\
\text { installed }\end{array}$ & $\sim 100 \mathrm{kWp}$ & Based on limited information of 3 projects. \\
\hline Number of projects & 5 implemented, 25 concepts & Based on information from the NVE report 2018. \\
\hline Total funds invested & N/A & \\
\hline
\end{tabular}

\section{Poland}

\begin{tabular}{|l|l|l|}
\hline Essential information & Quantitative estimate & Notes \\
\hline $\begin{array}{l}\text { Current total number of } \\
\text { initiatives }\end{array}$ & $\sim 112$ & $\begin{array}{l}45 \text { housing cooperatives, 66 energy clusters, } \\
1 \text { energy cooperative. Government prefers } \\
\text { energy clusters. }\end{array}$ \\
\hline
\end{tabular}




\begin{tabular}{|l|l|l|}
\hline $\begin{array}{l}\text { Historical evolution of number } \\
\text { of initiatives }\end{array}$ & Emerging sector. & Implementation of EU Directives ongoing. \\
\hline Field of engagement & $\begin{array}{l}\text { Solar PV, biogas, housing } \\
\text { cooperatives, energy clusters }\end{array}$ & Limited data. \\
\hline People involved & $\sim 784$ & Assuming minimum 7 members. \\
\hline Renewable capacities installed & $\begin{array}{l}\text { Housing PV: 0.045-1.8 MW } \\
\text { Energy clusters (PV, biogas, other): } \\
150 \text { MW }\end{array}$ & $\begin{array}{l}\text { From Schwanitz et al. 2021. Assuming average } \\
\text { apartment/ house installations of 10-40 kW. }\end{array}$ \\
\hline Number of projects & At least 112. & Assuming a project per initiative. \\
\hline Total funds invested & $\mathrm{n} / \mathrm{a}$ & \\
\hline
\end{tabular}

\section{Slovenia}

\begin{tabular}{|l|l|l|}
\hline Essential information & Quantitative estimate & Notes \\
\hline $\begin{array}{l}\text { Current total number of } \\
\text { initiatives }\end{array}$ & 7 & $\begin{array}{l}\text { COMETS database. Common legal form: } \\
\text { limited liability coop (z.o.o.). }\end{array}$ \\
\hline $\begin{array}{l}\text { Historical evolution of number } \\
\text { of initiatives }\end{array}$ & $\mathrm{n} / \mathrm{a}$ & Recent developments, still in their infancy. \\
\hline Field of engagement & Solar PV $(\sim 100 \%)$, wind & Recent activities. \\
\hline Number of people & $\mathrm{n} / \mathrm{a}$ & \\
\hline Renewable capacities installed & $\sim 60 \mathrm{~kW}$ & Based on the number of total kW/prod. units. \\
\hline Number of projects & 7 & Assuming at least 1 project/CAl. \\
\hline Total funds invested & $\mathrm{n} / \mathrm{a}$ & \\
\hline
\end{tabular}

\section{Sweden}

\begin{tabular}{|l|l|l|}
\hline Essential information & Quantitative estimate & Notes \\
\hline $\begin{array}{l}\text { Current total number of } \\
\text { initiatives }\end{array}$ & $\begin{array}{l}\text { 312 CAls (incl. liquidated, 10\%). } \\
\text { Additionally, 32 ecovillages. }\end{array}$ & $\begin{array}{l}\text { COMETS database. Number for eco-villages } \\
\text { from Magnusson \& Palm 2019. Legal forms: } \\
\text { different types of associations/coops. }\end{array}$ \\
\hline $\begin{array}{l}\text { Historical evolution of number } \\
\text { of initiatives }\end{array}$ & $\begin{array}{l}1990-2010 \text { expansion phase, boom } \\
\text { in wind coops. Since 2015 growth in } \\
\text { solar PV. }\end{array}$ & $\begin{array}{l}\text { Favourable legislation (e.g., high CO2 tax, RE } \\
\text { subsidies). Change in taxes in 2009 led to a } \\
\text { halt in wind coop and retreat of investors. }\end{array}$ \\
\hline Field of engagement & $\begin{array}{l}\text { Wind (38\%), solar PV (10\%), hydro } \\
(7 \%), \text { electricity trading (13\%), } \\
\text { heat/wood (11\%), distribution (10\%) }\end{array}$ & $\begin{array}{l}\text { Housing cooperatives often engage in } \\
\text { electricity trading (consumption). }\end{array}$ \\
\hline Number of people involved & $\begin{array}{l}\text { 20.08-0.1 million (2018). One coop } \\
\text { with 52000, smallest with 20 } \\
\text { members. }\end{array}$ & $\begin{array}{l}\text { Based on the sum of database entries. Does } \\
\text { not include eco-villages. }\end{array}$ \\
\hline Renewable capacities installed & $\begin{array}{l}\text { Solar PV: 1.2-13.1 MW, wind: } \\
153.8-244.6 \text { MW, hydro: } 6.4 \mathrm{MW}, \\
\text { other (biomass, solar thermal) up to } \\
2.9 \text { MW. Total capacity installed: } \\
\sim 165-267 M W .\end{array}$ & $\begin{array}{l}\text { Estimate based database (excluding } \\
\text { shares, 0\% (100\%) assumed if no information } \\
\text { available for lower (upper) estimate. }\end{array}$ \\
\hline
\end{tabular}




\begin{tabular}{|l|l|l|}
\hline Number of projects & $\begin{array}{l}\sim 311 \text { projects realized, out of which } \\
272 \text { are wind. }\end{array}$ & $\begin{array}{l}\text { Based on database entries. No information } \\
\text { about plans. }\end{array}$ \\
\hline Total funds invested & $\mathrm{n} / \mathrm{a}$ & \\
\hline
\end{tabular}

\section{Switzerland}

\begin{tabular}{|l|l|l|}
\hline Essential information & Quantitative estimate & Notes \\
\hline $\begin{array}{l}\text { Current total number of } \\
\text { initiatives }\end{array}$ & 291 & $\begin{array}{l}\text { Few more to Holstenkamp 2018. Typical legal } \\
\text { forms: traditional and modern cooperatives. }\end{array}$ \\
\hline $\begin{array}{l}\text { Historical evolution of number } \\
\text { of initiatives }\end{array}$ & $\begin{array}{l}\text { Steady development, three waves } \\
\text { since 1890' (2. 1980', 3. 2010'). }\end{array}$ & $\begin{array}{l}\text { Historic 'electra', recent ones often with 'solar' } \\
\text { or 'energy' in the name. Since 2016, more } \\
\text { often joint purchasing / do-it-yourself coops } \\
\text { have been founded. }\end{array}$ \\
\hline Field of engagement & $\begin{array}{l}\text { Solar PV (33\%), hydro (7-10 cases), } \\
\text { wind (1-3 cases), biogas (1), heat } \\
(19 \%), \text { distribution. }\end{array}$ & $\begin{array}{l}\text { About 20\% engage broader, i.e. beyond the } \\
\text { installation project. }\end{array}$ \\
\hline Number of people involved & $\begin{array}{l}137640, \text { thereof two bigger ones } \\
\text { with 67000 members. }\end{array}$ & $\begin{array}{l}\text { Typical size: 50-150 members. Based on } \\
\text { database entries. Traditional elektras in rural } \\
\text { areas. }\end{array}$ \\
\hline Renewable capacities installed & Upcoming. & $\begin{array}{l}\text { Installations with 50-100 kW (most), up to 350 } \\
\text { kW (few), } 1000 \text { kW (few). Method based on } \\
\text { rule of thumb to convert: Solar: 1000 } \\
\text { kWh/kWp. wind: 30-40\% efficiency x 8640 for } \\
\text { kWh; hydro: 8000. }\end{array}$ \\
\hline $\begin{array}{l}\text { Number of projects } \\
\text { administered }\end{array}$ & $\begin{array}{l}\text { 1200-1500 } \\
\text { Total funds invested }\end{array}$ & $\begin{array}{l}\text { Typically, 4-5 projects per CAl. Estimate based } \\
\text { on CAl number. }\end{array}$ \\
\hline \begin{tabular}{l} 
Estimate based on extrapolation to all. \\
\hline
\end{tabular}
\end{tabular}

\section{Energy communities/collective action initiatives in the energy transition:}

Bulgaria: High financial and administrative barriers to start citizen-led initiatives. An interesting example is the attempt to explore options for forming a cross-border coop between Burgas in Bulgaria and Nis in Serbia to co-own PV systems (supported by H2020 project mPower, 2018-2022). Alleviation of energy poverty is an important motivation for establishing community energy initiatives.

Cyprus: Currently no initiative. However, the implementation of EU Directives is on its way and it is expected that a few energy communities are established soon (Cyprus Energy Agency 2021). Consulting material (in Greek language) is available to support potential initiatives.

Greece: Under construction. Unclear what forms qualify as CAI. About 50 candidates.

Hungary: Two community projects identified: 1 ecovillage (biomass) and 1 crowdfunding PV project (planning stage, $30 \mathrm{kWp}$ ). Administrative and technical barriers towards financial sustainability. Legislative barriers for wind energy $(12 \mathrm{~km}$ from settlement rule, banning almost the whole country) and community energy as a whole as prosumers are not allowed to be energy suppliers (electricity has to be sold to grid with low revenue). Strong focus on nuclear power development in cooperation with Russia. EU frameworks are implemented with the minimum requirements (in progress). Little information available. 


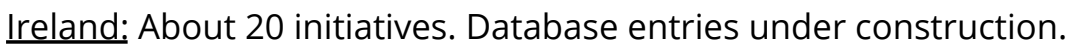

Latvia: 3 housing associations. They renovate heat pump systems, improving energy efficiency through reduction by about 50\%. Implementation of EU Directives ongoing.

Liechtenstein: Under construction.

Malta: One active RE cooperative, but information is limited. The first national, communal PV farm has started operation: $997 \mathrm{kWp}, 366$ crowdfunding applicants to provide rooftops were accepted to the project (Energy and water agency Malta 2021). The requirement for a start capital of 0.1 million EUR is seen as a barrier (Cooperatives Europe 2015).

Portugal: Under construction. 1 CAI known.

Romania: High legal and financial barriers for citizens to engage. High centralization of legislative and executive powers makes it difficult for local parties to participate. State of Implementation of EU Directives. One cooperative was established in 2019.

Slovakia: Implementation of EU Directives ongoing. High financial and administrative barriers. Negative investment experiences from the 1990 still hamper interest from citizens.

Spain: About 20 CAls. Entries under construction.

\section{Summary}

We present preliminary estimates for the contribution of collective prosumers to the energy transition across Europe. Drawing from the COMETS inventory, we report country-level and EU-level aggregates. Aggregating to the European level, we estimate that:

- Currently, at least two million citizens across Europe collectively engage in at least 8431 energy communities that have their primary focus on the energy sector, realizing a minimum of 13000 projects (since 2000).

- Differences across countries can be explained by a) the strength of governmental support and incentives schemes, b) historic path dependencies of the energy system, and c) social and technological preferences.

- Current total renewable capacities installed by citizen-led energy projects in Europe can be estimated at least as high as $6.3 \mathrm{GW}$, contributing up to $7 \%$ to the nationally installed capacities. Solar PV contributes half of the capacities, followed by onshore wind (10\%).

- A conservative estimate of the total invested finances amounts to 2.6 billion EUR.

\section{Acknowledgements}

This research was funded by the European Union's Horizon 2020 research and innovation programme under grant agreement No 837722, project COMETS (COllective action Models for Energy Transition and Social Innovation). The funding source was not involved in the study.

\section{References}

Berka, A.L., \& Creamer, E. (2018) Taking stock of the local impacts of community owned renewable energy: A review and research agenda. Renewable \& Sustainable Energy Reviews, 82, 3400-3419.

Boon, F.P., \& Dieperink, C. (2014) Local civil society based renewable energy organisations in the Netherlands: Exploring the factors that stimulate their emergence and development, Energy 
Policy, 69, 297-307. Local civil society based renewable energy organisations in the Netherlands: Exploring the factors that stimulate their emergence and development

Co2mmunity (2021). Database of community energy projects in the BSR. Retrieved from http://old.lei.It/co2mmunity/?s=\&category=\&location=16\&a=true

Coppedge, Michael, John Gerring, Carl Henrik Knutsen, Staffan I. Lindberg, Jan Teorell, Nazifa Alizada, David Altman, Michael Bernhard, Agnes Cornell, M. Steven Fish, Lisa Gastaldi, Haakon Gjerløw, Adam Glynn, Allen Hicken, Garry Hindle, Nina Ilchenko, Joshua Krusell, Anna Luhrmann, Seraphine F. Maerz, Kyle L. Marquardt, Kelly McMann, Valeriya Mechkova, Juraj Medzihorsky, Pamela Paxton, Daniel Pemstein, Josefine Pernes, Johannes von Römer, Brigitte Seim, Rachel Sigman, Svend-Erik Skaaning, Jeffrey Staton, Aksel Sundström, Ei-tan Tzelgov, Yi-ting Wang, Tore Wig, Steven Wilson and Daniel Ziblatt. 2021. "V-Dem [Country-Year/Country-Date] Dataset v11.1" Varieties of Democracy Project. https://doi.org/10.23696/vdemds21

Cooperatives Europe (2015). Cooperatives Europe key figures 2015. Retrieved from https://coopseurope.coop/sites/default/files/The\%20power\%20of\%20Cooperation\%20-\%20Coop eratives\%20Europe $\% 20$ key\%20statistics\%202015.pdf

Energy and water agency Malta (2021). Other energy projects - Communal Photovoltaic Farm. Retrieved from https://www.energywateragency.gov.mt/other-energy-projects/

Hier Opgewekt (2021). Hier Opgewekt - Lokale Energie Monitor. Retrieved from https://www.hieropgewekt.nl/

Hufen, J.A.M. , Koppenjan, J.F.M (2015) Local renewable energy cooperatives: revolution in disguise? Energy, Sustainability and Society, 5 (1). https://doi.org/10.1186/s13705-015-0046-8

Madlener, R., Glensk, B., Gläsel, L. (2019). Optimal Timing of Onshore Wind Repowering in Germany under Policy Regime Changes: A Real Options Analysis. Energies 2019, 12: 4703. https://doi.org/10.3390/en12244703

Magnusson, D. \& Palm, J. (2019). Come Together-The Development of Swedish Energy Communities. Sustainability, 11(4), 1056. https://doi.org/10.3390/su11041056

Milieu Centraal (2021). Kosten en opbrengst zonnepanelen. Retrieved from https://www.milieucentraal.nl/energie-besparen/zonnepanelen/kosten-en-opbrengst-zonnepane len/\#Zonnepanelen-worden-steeds-goedkoper

Norges Vassdrag og Energi Direktorat (NVE) (13 December, 2018). Descriptive study of Local Energy Communities. THEMA Report 2018-20. Retrieved from: https://thema.no/wp-content/uploads/THEMA-Reort-2018-20-Local-Energy-Communities-ReportFinal.pdf

Paardenkooper, M. (2015). Feasibility of onshore wind energy in the province of North-Holland, Master Thesis, VU University Amsterdam.

RESCOOP (July 2021). July success story: the beginning of community energy in Romania. Retrieved from: https://www.rescoop.eu/news-and-events/news/july-success-story-the-beginning-of-communityenergy-in-romania 
Schwanitz, V. J., Rudek, T., Hubert, W., Wierling, A. [Under review] (2021). The development of citizen-installed renewable energy capacities in former Eastern Bloc countries - The case of Poland.

Von Beck, C. (2021). Citizen-led initiatives in the German wind energy sector - a qualitative and quantitative exploration, Master's Thesis, Western Norway University of Applied Science.

Wierling, A., Zeiss, J. P., Lupi, V., Candelise, C., Sciullo, A. and Schwanitz, V.J. (2021). The Contribution of Energy Communities to the Upscaling of Photovoltaics in Germany and Italy. Energies 14, no. 8: 2258.

Wierling, A., Zeiss, J. P., von Beck, C. and Schwanitz, V.J. [Under review] (2021 b). Business models of energy cooperatives active in the PV sector - A statistical analysis for Germany.

Wierling, A., Schwanitz, V.J., Gregg, J., Zeiss, J. P., Bout, C., Candelise, C. and Gilcrease, W. (2018). Statistical evidence on the role of energy cooperatives for the energy transition in European countries Sustainability 10: 3339. https://doi.org/10.3390/su10093339

Wierling, A., Zeiss, J. P. , Hubert, W., Candelise, C., Gregg, J. and Schwanitz, V. J. (2020). Who participates in and drives collective action initiatives for a low carbon energy transition? in Nedelciu, C., Oostdijk, M., Morales, M., Schellens, M. and Diemer, A. (eds.) Paradigms, models, scenarios and practices for strong sustainability, Editions Oeconomia. 1. 